Research Paper

\title{
Hypothalamic beta-endorphin neurons suppress preneoplastic and neoplastic lesions development in 1,2-dimethylhydrazine induced rat colon cancer model
}

\author{
Sengottuvelan Murugan², Yatee Dave ${ }^{1}$, Ankush Rakhit ${ }^{1}$, Dipak. K. Sarkar ${ }^{1 凶}$ \\ 1. Endocrine Program, Department of Animal Sciences, Rutgers, The State University of New Jersey, New Brunswick, New Jersey 08901, USA; \\ 2. Current address: Perelman School of Medicine, University of Pennsylvania, Philadelphia, PA 19104, USA. \\ $\triangle$ Corresponding author: Dr. Dipak K. Sarkar, Distinguished Professor and Director, Endocrine Program, The State University of New Jersey, Endocrine \\ Research Facility, 67 Poultry Farm Lane, New Brunswick, NJ 08901, USA. Phone: 848-932-1529; Fax: 732-932-4134; e-mail: sarkar@aesop.rutgers.edu \\ (C) Ivyspring International Publisher. This is an open access article distributed under the terms of the Creative Commons Attribution (CC BY-NC) license \\ (https://creativecommons.org/licenses/by-nc/4.0/). See http://ivyspring.com/terms for full terms and conditions.
}

Received: 2016.12.21; Accepted: 2017.08.29; Published: 2017.09.06

\begin{abstract}
In recent years, experimental studies demonstrated negative impacts of impaired body stress response on colonic pathologies. In this study, we tested if reducing body stress response by the use of $\beta$-endorphin (BEP) neuronal transplants in the hypothalamus suppresses pre-neoplastic and neoplastic lesions. Colon cancer was induced by injecting 1,2-dimethylhydrazine (DMH) for sixteen weeks in Sprague Dawley rats with BEP neuron transplants or control neuron transplants, and their colonic histopathologies, colon tissue levels of pro-inflammatory cytokines and epithelial-mesenchymal transition (EMT) proteins and splenic levels of cytotoxic proteins were measured. Our results revealed that DMH induced tumors in colon at $100 \%$ incidence in control rats but failed to induce colonic tumors in $70 \%$ of animal with BEP neuronal transplants. The mean volume of tumor at the colon was smaller in BEP neurons transplanted rats than those in controls. Histopathologies of colon tissues revealed that BEP neurons transplanted animals had lesser tissue lesions such as aberrant crypt foci (ACF) and adenocarcinoma development in the colon than those in control groups. Immunohistochemical and western blot analyses identified reduced expression of Ki-67, TNF- $\alpha$ and NF-kB nuclear translocation in colonic tissues of BEP neurons transplanted rats than those in controls. BEP neurons transplanted rats also showed reduced expressions of transcription factors linked to EMT like Snail, Twist, and N-cadherin, but increased the levels of an epithelial cell marker E-cadherin in colon tissue. Furthermore, splenic NK cells cytolytic proteins such as perforin, granzyme B and IFN- $\gamma$ levels in BEP neurons transplanted rats were higher than those in control rats. These data suggest that BEP neuron transplants suppress the growth and progression of colonic tumors possibly by decreasing inflammatory mileu and EMT via activation of innate immune responses.
\end{abstract}

\section{Introduction}

Colorectal cancer (CRC) is the second most diagnosed cancer with high mortality that affect both men and women in the united states (1). Based on the epidemiological studies, it has been estimated that as much as $70-80 \%$ of CRC could be attributed to the contribution of environmental and lifestyle factors (2). A number of studies now identified that increased amount of body stress such as psychosocial challenges, physiological, hormonal and immunological imbalances increases the risk of cancer
(3). Also, reduction of body stress via the method of increasing optimistic attitude has been shown to help cancer patients extend their longevity (4). As a result of such findings, therapies have been developed to target the body's mechanisms to handle stress, in an attempt to indirectly target cancer.

Hypothalamic proopiomelanocortin neurons produce various peptides including $\beta$-endorphin (BEP) which is an endogenous opioid peptide that promotes feelings of well-being and plays a key role 
in maintaining body stress response via inhibitory control of the hypothalamic-pituitary-adrenal axis function and the sympathetic neuronal activity (5). Therefore, it has the potential to inhibit and reduce stress behavior and stress associated imbalances. Manipulations of BEP neuronal activities in the hypothalamus have been conducted in animal models that have been induced to have cancer, and the subsequent effect on the animal cancer growth was observed. BEP neuronal activation was found to inhibit mammary, prostate and liver tumor cell growth and inhibit these cancer cells metastasis (6-9). The cancer preventive effect of BEP neuron is caused by the suppression of sympathetic neuronal function and resulting in an increased peripheral NK cell and macrophage activities, elevated levels of anti-inflammatory cytokines and reduced levels of inflammatory cytokines. BEP neuronal inhibitory effects on tumor progression also involves alteration in tumor microenvironment possibly due to suppression of catecholamines and inflammatory cytokines production that are known to alter cell-matrix attachments, angiogenic mechanisms, DNA repair and epithelial-mesenchymal transition (6, 10-12). It is not yet known whether BEP neuronal transplants have preventive effects on the growth and metastasis of colon cancer. In this study we evaluate the cancer preventive effect of BEP neuron transplantation on $\mathrm{DMH}$-induced colon cancer in rats.

\section{Materials and Methods}

\section{Preparation of BEP cells from neural stem cells}

We isolated neural stem cells from 17 days old fetal rat brains of Sprague Dawley rats and then differentiated these cells into BEP neurons in vivo. We used cAMP and pituitary adenylate cyclase-activating polypeptide (PACAP) to differentiate BEP neurons from rat fetal neural stem cells, as previously described (9).

\section{Transplantation of BEP neurons into the hypothalamus of rats}

An animal model was utilized to investigate the effects of BEP neuron transplantation on the development of colon cancer. Adult male Sprague-Dawley rats (150-200 g; 60 days of age) were fed water and a chow ad libitum diet daily throughout the study. Animals were stereotaxically injected with 20,000 BEP neurons or control neurons in each paraventricular nucleus (PVN) of the hypothalamus. Cortical neurons served as control since these neurons do not secret BEP. Animal surgery and care were performed in accordance with institutional guidelines and complied with the National Institutes of Health policy. The animal protocol (99-005) was approved by Rutgers Animal Care and Facilities Committee.

\section{Induction of colon cancer with colon specific carcinogen}

Two weeks after the neuronal transplantation, rats were injected with a colon specific carcinogen, 1,2-dimethylhydrazine dihydrochloride $(\mathrm{DMH})$, at a dosage of $15 \mathrm{mg} / \mathrm{kg}$ body weight (13-15). Rats were exposed to carcinogen twice weekly, via subcutaneous administration, for 15 weeks. The DMH was dissolved in $0.9 \%$ saline containing $1.5 \%$ EDTA and was adjusted to $\mathrm{pH} 6.5$ with $1 \mathrm{M}$ sodium hydroxide prior to injection. Observations of the animals were taken daily, and weight measurements were conducted weekly. Fifteen weeks after the last $\mathrm{DMH}$ injection rats were sacrificed by decapitation, and normal and cancerous colon tissue was collected and portion of distal colon was stored in 10\% neutral-buffed formalin for histology and the rest were snap frozen in liquid nitrogen and stored at $-80^{\circ} \mathrm{C}$ for Western blotting of various protein.

\section{Preparation for aberrant crypt foci counting}

The colon was processed as follows for the determination of aberrant crypt foci (ACF; 16). The entire colon (from caecum to anus) was removed and washed thoroughly with $0.9 \%(\mathrm{w} / \mathrm{v}) \mathrm{NaCl}$, cut longitudinally, laid flat on a polystyrene board and fixed with $10 \%(\mathrm{v} / \mathrm{v})$ buffered formaldehyde solution overnight. The colon was then stained with $0.2 \%$ $(\mathrm{w} / \mathrm{v})$ methylene blue for 2-3 min in saline in order to identify the ACF. Mucosal ACF were counted using a light microscope (40 $\mathrm{X}$ or $60 \mathrm{X}$ magnification). The ACF were classified as small (One to three crypts), medium (four to six crypts) or large (more than six crypts) by the number of crypts per foci. The total numbers of ACF were calculated as the sum of the small, medium and large ACF.

\section{Histopathological observation}

Formalin fixed colon tissues were embedded in paraffin and cut into $5-\mu \mathrm{m}$ sections and used for hematoxylin and eosin (H\&E) staining. The pathological changes of colon tissue were evaluated by a two investigators blinded to treatment as described previously (17).

\section{Western blotting}

For Western Blotting, colonic tumor tissue extracts $(50 \mu \mathrm{g})$ and Western blot chemiluminescence reagent (Thermo Fisher Scientific Inc., Rockford, IL) were used. Primary antibodies used were: anti-perforin rabbit polyclonal (1:250), anti-granzyme 
B mouse monoclonal (1:200), anti-IFN- $\gamma$ mouse monoclonal (1:250) and anti-COX-2 (1:200), Snail (1:200), Slug (1:200), and Twist (1: 250) all from Santa Cruz Biotechnology, Inc., (Dallas, TX) and anti-TNF-a (1: 200) and anti-NF-kB (1:300), E-cadherin (1:250) and $\mathrm{N}$-cadherin (1:250) were from (Abcam, Cambridge, MA). The densitometry was performed using Image J analysis software (National Institutes of Health, Bethesda, MD). Each protein was normalized with $\mathrm{b}$-actin or lamin B1 (nuclear).

\section{Immunohistochemical localization of proteins.}

Immunohistochemistry was performed on thin paraffin sections $(5 \mu \mathrm{m})$ of colon tissues mounted on Superfrost Plus glass slides (Fisher Scientific, Itasca, IL) and heated at $37^{\circ} \mathrm{C}$ overnight. The sections were deparaffinized in xylene, gradually hydrated with decreasing concentrations of ethanol and water, and endogenous peroxidases were removed with $0.3 \%$ $\mathrm{H}_{2} \mathrm{O}_{2}$ in methanol for $30 \mathrm{~min}$. Then the slides were subjected to antigen retrieval by microwave irradiation for $10 \mathrm{~min}$ at $98^{\circ} \mathrm{C}$ in $0.1 \mathrm{M}$ citrate buffer (pH 6.0). After cooling, the slides were incubated with a blocking reagent $(2 \% \mathrm{BSA}$ in $\mathrm{PBS})$ at $25^{\circ} \mathrm{C}$ for $30 \mathrm{~min}$ and subsequently incubated overnight at $4^{\circ} \mathrm{C}$ with primary antibodies. Primary antibodies for immunohistochemistry were used as follows: polyclonal rabbit antibodies against b-catenin (1:100; Cell Signaling Technology Inc., Danvers, MA) and tissue culture supernatant of the mouse monoclonal antibody for Ki-67 (1:50; from Abcam, Cambridge, MA). After the primary antibody incubation and PBS wash, sections were incubated with peroxidase-coupled anti-rabbit or anti-mouse IgG as secondary antibodies (ImmPRESS reagent, Vector Laboratories, Inc., Burlingame, CA). Antigen localization was achieved by using the 3,3'-diaminobenzidine-peroxidase reaction and sections were stained with Gill'\#3 hematoxylin (1:4) as a blue nuclear counterstain, dehydrated, and cover slipped with Permount (Fisher Scientific, Fair Lawn, NJ). To evaluate the immunohistochemical staining, five animals in each experimental group and five microscopic fields per animal were photographed using Nikon-TE 2000 inverted microscope. Intensity of staining was categorized as negative $(-)$, weak $(+)$, moderate $(++)$ and strongly positive $(+++)$.

\section{Statistical analysis}

Statistical analysis was performed by using GraphPad Prism 5 software (Graph Pad Software Inc., San Diego, CA). The data represented in figures are mean and SEM of 10 independent colon samples of rats from each treatment. Differences between the groups were analyzed using one-way ANOVA with the Newman-Kuel posttest. A value of $\mathrm{P}<0.05$ was considered to be significant.

\section{Results}

Male rats with neuronal transplants and given weekly DMH injections for 15 weeks were inspected for gross appearance of colon tumors. As shown in Fig, 1A and B, gross appearance of colon showing large multiple tumors in cortical neurons transplanted animals but show a small size tumor in BEP neuronal treated animals. ACF are pre-neoplastic lesions that serve as biomarkers for colon cancer $(15,18)$. Aberrant crypts stain heavily with methylene blue and are larger than normal crypts and have characteristic elliptical shape and thickened epithelial lining (Fig. $1 C, D)$. They are characterized in size by the number of abnormal crypts within each cluster. A greater number of larger sizes ACF correlate to a greater chance for colon cancer development. Results revealed that BEP neuron-transplanted animals had lesser small, medium and large ACF and overall total number of ACF (Fig. 1D, E), than the control group (Fig. 1C, E). This suggests that BEP implantation suppressed pre-neoplastic lesion development in the colon when compared to cortical cell-transplanted animals.

Administration of DMH resulted in tumorigenesis in the two experimental groups but at different incidence. Figure $1 \mathrm{~F}$ shows that the BEP-treated group had significantly lower tumor incidence $(30 \%)$ than the control group $(100 \%)$. The mean volume of tumors in the colon was smaller in BEP neuron-transplanted rats than in control cell-transplanted rats (Fig. 1G), which reveals the tumor suppressive effect of $\beta$-endorphin cells. In addition, the histopathological examination analysis of tumor phenotype reveals that BEP neuron-transplanted animals had adenomas, which are benign tumors of the epithelial tissue (Fig. 1H, I). In contrast, the control group had mostly adenocarcinomas $(70 \%)$ in the colon. The latter is malignant.

The fundamental etiology for carcinogenesis is uncontrolled cell proliferation. Consequently, any factor that can inhibit abnormal cell growth has potential to inhibit cancer development $(19,20)$. In order to evaluate the ability of BEP to modulate cell proliferation, the cell proliferation marker ki-67 was screened in the tumor of both groups. Immunostaining of ki-67 reveals that there was more ki-67 expression in cells from the control groups (Figure 2B, D \& E) than cells from the BEP-treatment group (Figure 2A, $C \& E$ ). This difference indicates that $\mathrm{BEP}$ has a potential effect to hinder cell 
proliferation, and by extension, carcinogenesis.

Beta-catenin plays a fundamental role in the regulation of the E-cadherin-catenin cell adhesion complex. Alterations of beta-catenin expression, in addition to its binding partners E-cadherin and the adenomatous polyposis coli protein (APC), are frequent events in colorectal cancer $(3,21)$. Immunostaining of beta-catenin shows that there was lack of expression (Figure 3A) predominantly limited to the lateral cell membrane in colonic epithelium cells in the BEP neuron-transplanted group (Figure 3C), where beta-catenin is normally expressed. Conversely, beta-catenin expression is aberrant (Figure 3B) and mostly translocated into the nucleus of colonic epithelium cells (Figure 3D) in the cortical cell group, which reflects the more malignant phenotype in the control group.

Chronic inflammation has been implicated in having an association with carcinogenesis. The constant presence of circulating inflammatory cells induces a microenvironment that facilitates tumorigenesis (22-25). We investigated several
Cortical Cells
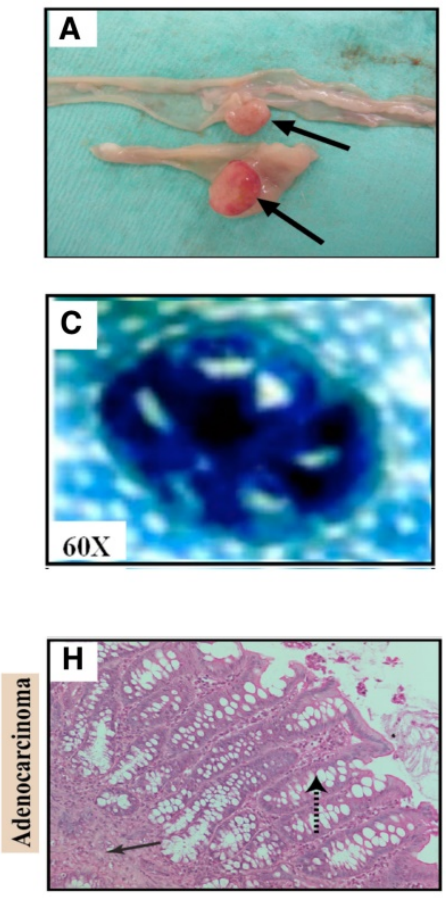

BEP Cells
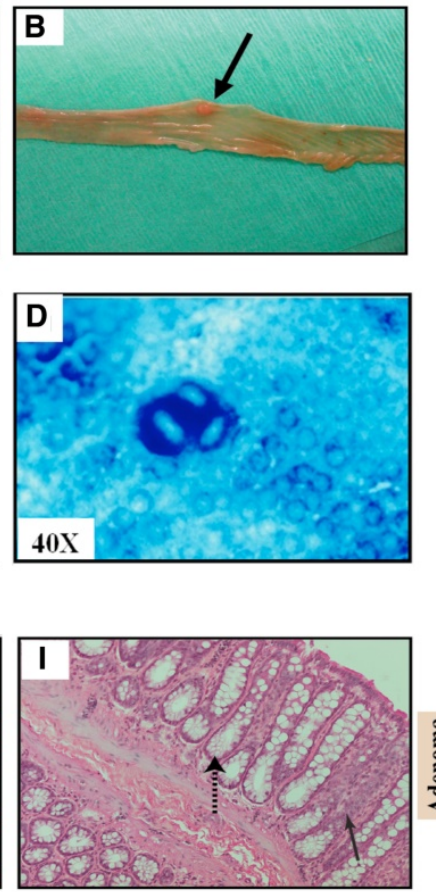

Figure 1. Effects of BEP neuron transplants in the hypothalamus on DMH-induced histopathologies in the colon. (A, B) Gross appearances of colon tissues showing by arrows large multiple tumors in cortical cells transplanted animals (A) and small tumor in BEP cells transplanted animals (B). Images are at $2 x$. (C, D) Representative pictures of aberrant crypt foci stained using methylene blue in colonic tumor in control group (C; magnification 60x) but small ACF in tumors of BEP neurons transplanted group (D; magnification 40x). (E) Incidence of aberrant crypt foci (ACF) in different sizes (small, medium, large) and total numbers in BEP cell transplanted rats and control rats are shown in histograms. (F, G) Mean and SEM percentage of tumor incidence $(F)$ and volume $(G)$ in cortical cells and BEP cells transplanted rats are shown in histogram panels. Data are presented as mean and SEM $(n=10)$. $* P<0.05$ and $* * * P<0.001$ between BEP cells and cortical cells transplanted groups. $(H, I)$ Representative photomicrographs of H\&E-stained paraffin sections of the control $(\mathrm{H})$ and $\beta$-endorphin cells transplanted groups (I) at 20X magnification. Colonic tumor of control rat showing adenocarcinoma (solid arrow) with irregular crypt structures (broken arrow) $(\mathrm{H})$. BEP cells implanted rats show adenomas (solid arrow, benign) with normal crypt structures (broken arrow; I).

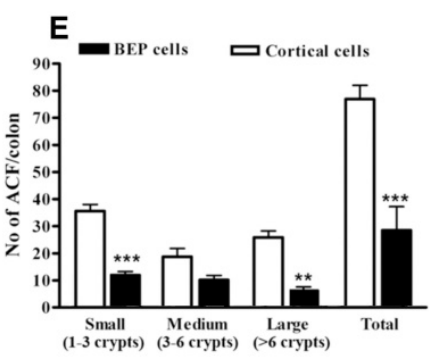

$\mathbf{F}$

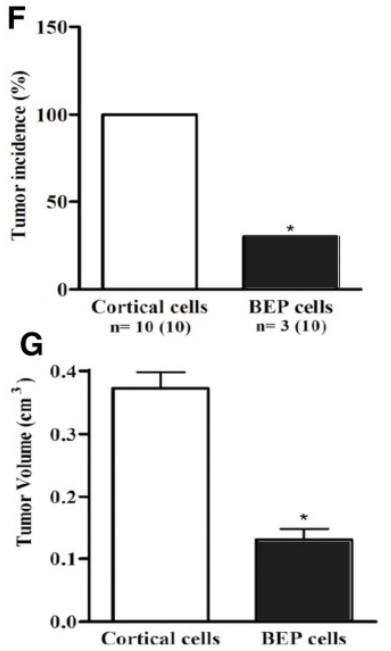

inflammatory proteins to determine whether treatment with beta-endorphin would lead to an atement of chronic inflammation. Western blotting inflammatory markers TNF-a, COX-2, and NF-kB ere expressed respectively in the control group, inflammation in colon tissue.

Increased epithelial-mesenchymal transition is associated with an increased incidence of cancer, as well as a greater potential for metastasis (26-28). The ent of EMT occurrence can be determined by experimentation. Western blotting was used to detect the expression levels of Snail, Twist, N-cadherin, and E-cadherin. There was overexpression of Snail, Twist, and $\mathrm{N}$-cadherin in cortical cells from rats that were induced with cancer, but not treated with BEP neuronal transplant. This is evident in Figure 5A, B and D. Tissues from rats exposed to BEP therapy had higher E-cadherin

expression, when compared with the control, as represented by Figure 5C. These results suggest BEP plays a role in regulation of EMT.

The primary defense cells of the innate immune system participate in various pathways to attack cancer cells (29-32). NK cell activity can be monitored via measurement of $\mathrm{NK}$ cell-mediated factors, such as granzyme-B, perforin and IFN- $\gamma$. 
Western blotting was used to measure expression from spleen tissue. There was upregulation of NK cell cytotoxic protein expression (granzyme, perforin and IFN-gamma) in BEP-treated groups, as demonstrated by Figure 6A, B and $\mathrm{C}$ respectively. Control groups had less protein expression. This evidence suggests that beta-endorphin has an effect on NK cell activity.

\section{Discussion}

Colorectal cancer incidence has been decreasing over the years, but remains the second most cancer with high mortality $(1,2)$. Understanding the factors and processes that influence the colon and its development can elucidate some of the ways in which colorectal cancer can be prevented. As the results indicate, BEP treatment has proven to be effective at various points of the process in which chronic inflammation and chronic stress lead to cancer. In terms of physical analysis, both experimental groups had evidence for tumorigenesis in the colorectal region. The reduced incidence and less malignant nature of the tumors of the BEP-treated group in contrast to the control group indicate the potential of $\mathrm{BEP}$ to counter cancer-promoting processes. Additional data provide evidence that BEP's anti-cancer effect is its ability to reduce the number and size of ACF. This indicates that crypts in the colon are developing normally in response to BEP exposure, despite induction by the $\mathrm{DMH}$ carcinogen. If BEP has
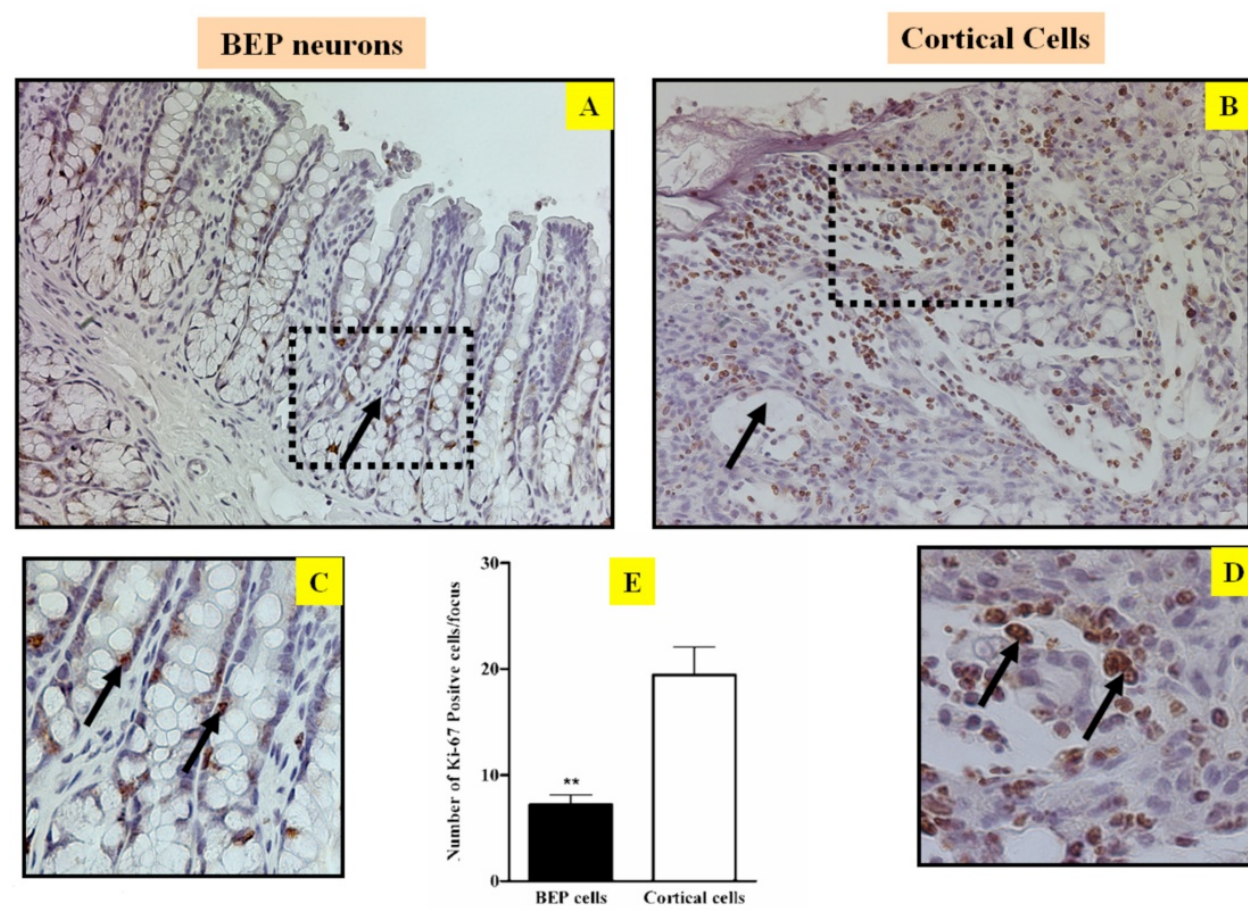

Figure 2. Effects of BEP neuron transplants in the hypothalamus on DMH-induced changes in cell growth marker Ki67 in the colon. Representative photomicrographs showing immunolabeled Ki-67 cells in colon of BEP (A, C) and cortical (B, D) neurons transplanted animals. Brown-stained spots are immunopositive cells. Images are at 20x magnification (A, B) except those inside boxes that are at 40x. (C, D).

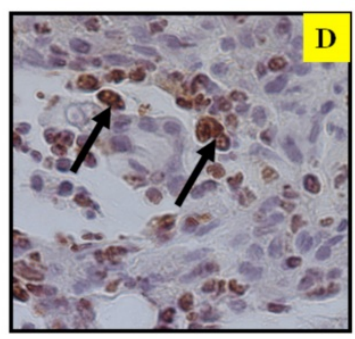

the ability to maintain normal colon development in the presence of carcinogens, it has the potential to be an even more effective preventative agent.

Analysis of the effects of BEP on biochemical factors involved with inflammation, innate immunity, cell proliferation, and epithelial-mesenchymal transition provide further evidence for the widespread role of BEP. A known instigator of deleterious responses within the body is chronic inflammation (22-24). Our results showed that there was a difference in inflammatory marker expression in colon tissue between the two groups. In contrast to the control groups, BEP neuronal therapy reduced the expression of inflammatory markers TNF-a, NF-kB, and COX-2. These results imply that inflammation accompanies and has some role in the progression of colorectal cancer. Because there was higher expression of inflammatory markers in the control group, which was found to have much higher incidences of adenocarcinomas, it is possible that in certain situations, inflammation can act as a tumor-promoter. Additionally, BEP appears to have a relationship with inflammatory processes that result in their ultimate inhibition. The potential of BEP to reduce the inflammatory response has significant implications in dampening the cascade that occurs as a consequence of chronic inflammation.

If persistent inflammation is inhibited, the sympathetic nervous system is no longer overstimulated. There are two consequences to this. One is that the colon is not innervated in excess, and therefore, can develop more normally. This would lead to decreased incidence of abnormal crypt development. Reduction in ACF was observed as a result of BEP neuronal therapy. Therefore, BEP neurons from the hypothalamus may have an effect on the colon via neural input of the sympathetic nervous system (5).

Second, BEP treatment was found to upregulate expression of NK cell-mediated cytotoxic factors perforin, granzyme-B, and IFN- $\gamma$. This is 
demonstrative of increased innate immune activity. NK cells carry out their function by activating cascades and secreting factors that mediate cellular apoptosis. BEP appears to have regulatory behavior that promotes immunity, possibly as a result of upstream effects of reducing chronic stress (5). Without inhibition by cortisol, the innate immune system can function properly and better conduct immunosurveillance of cancer cells. Manipulation of NK cell activity has great potential as an anti-cancer approach (30). Using BEP to maintain high NK cell activity in conjunction with alternate methods that manipulate NK cell-related factors can result in a potent tool with which to manage cancer.

If apoptosis were occurring as it should be against the proper cells, cell proliferation would be better controlled. The difference between a cancer cell and a normal cell is that the former is not regulated by the normal cell cycle. The aberrant regulation of these cells leads to unhindered growth. Inhibiting excessive proliferation can also stunt cancer growth and development. Colon crypt cells from the BEP-treated groups had less ki-67 expression than that of cells from the control group. Because epithelial cells in the colon have a relatively quick turnover rate, there would be some level of ki-67 expression in all colon tissue. However, those areas with the most expression

BEP neurons
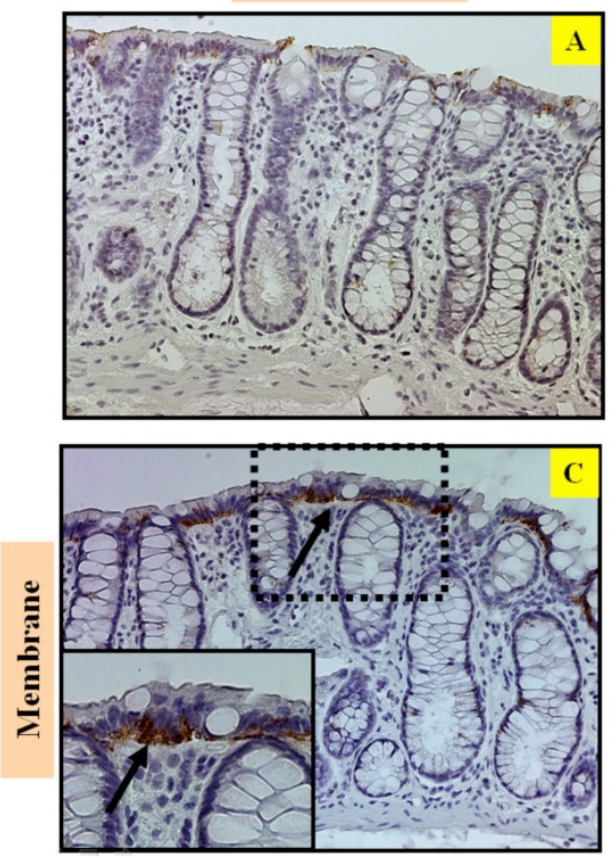

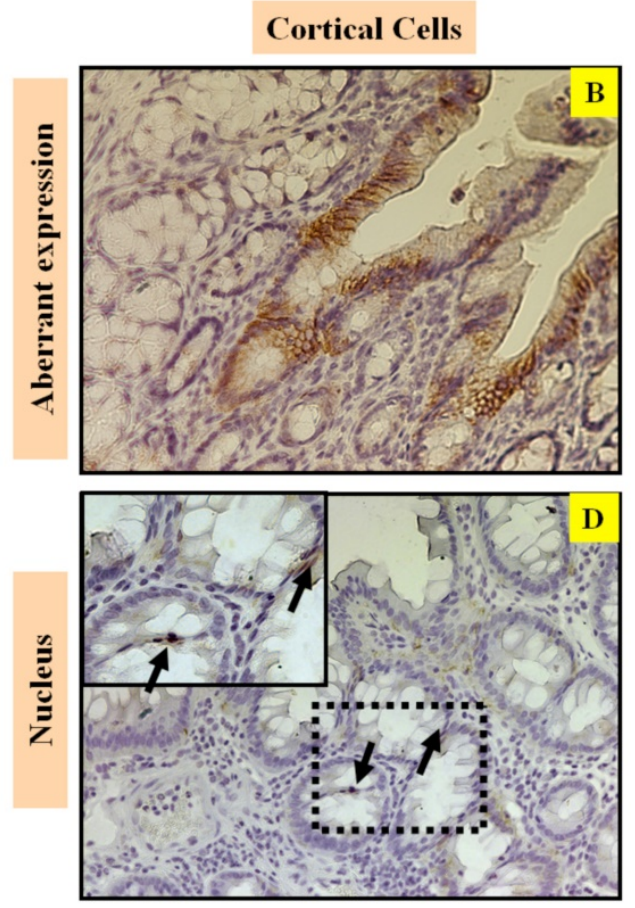

Figure 3. Effects of BEP neuron transplants in the hypothalamus on DMH-induced in Immunostaining of beta-catenin in the colon. Representative photomicrographs showing immunolabeled beta-catenin cells in colon of BEP (A, C) and cortical (B, D) neurons transplanted animals. Brown-stained spots are immunopositive cells. Images are at 20x magnification (A, B) except those inside boxes that are at 40x. (C, D). BEP neuron-transplanted group shows beta-catenin staining in lower intensity (A) and predominantly limited to the lateral membrane of colonic epithelium cells (C). Whereas, beta-catenin staining is aberrant (B) and translocated into the nucleus of colonic epithelium cells (D) in the cortical cell group, which reflects the more malignant phenotype in the control group. are predisposed to and more likely to exhibit carcinogenesis. Beta-endorphin seems to limit the amount of aberrant cell proliferation occurring, either through indirect or direct means.

By controlling cell proliferation, BEP also has the potential to reduce the incidence of EMT. As epithelial cells gain migratory properties, they increase the chances for metastasis. BEP-treatment reduced the expression of markers associated with EMT relative to the expression in the control groups. E-cadherin was downregulated while $\mathrm{N}$-cadherin was upregulated. This indicates that epithelial cells are maintaining their stationary status. Thus, even if there are tumor cells present there is less potential for them to become migratory and metastasize to other areas of the body. Since metastasis marks an extreme and often unrecoverable phase of cancer progression, being able to reduce the chances of such an event can greatly aid in reducing cancer severity.

Beta-catenin plays a fundamental role in the regulation of the E-cadherin-catenin cell adhesion complex (21). It also functions in growth signaling events, independently of the cadherin-catenin complex, and these signaling pathways are disturbed in colorectal cancer. Alterations of beta-catenin expression, and its binding partners E-cadherin and the adenomatous polyposis coli protein (APC), are frequent events in colorectal cancer $(3,21)$. Beta-catenin and E-cadherin expression were predominantly limited to the lateral cell membrane in normal colonic epithelium. Abnormal beta- catenin expression and/or shift from membranous to accumulation into cytoplasmic and nuclear localization are associated with malignant transformation. Beta-catenin is also associated with EMT. Disassociation of beta-catenin from E-cadherin is an indicator of EMT. Increased levels of free beta-catenin are demonstrative of a loss of cell adhesion. Just as an increase of migratory status can elevate the 
risk of metastasis, lack of adhesion can lead to a higher potential of metastasis. There was less beta-catenin expression in the BEP-treatment group than in the control group. Beta-catenin can potentially be assigned as a tumor-promoter because it was higher in the control group, which had a higher incidence of adenocarcinomas.

Stressful situations have become increasingly common in daily activities, but its prevalence in no way reduces the deleterious effects it can have on the body. Both neurological and behavioral stress can promote carcinogenesis, as well as tumor development by disrupting the balance of inflammatory markers, various cytokines, and catecholamines $(5,32-34)$. These factors create a tumor microenvironment that supports development of the tumor. Abnormal crypt development and cell proliferation result from a decrease in immunosurveillance and increase in migratory status of epithelial cells (35).

Part of the body's natural way in which to combat all this is via endorphins. Situations that induce stress, fear, or pain stimulate the secretion of endorphins, which act as endogenous opiates (5). The results detailed in this paper have demonstrated that BEP can have effects surpassing that of mere pain modulation. BEP can lessen inflammation and consequently, decrease the stress response. Doing so results in a stronger immune system that is better equipped to regulate proper cell proliferation and EMT.
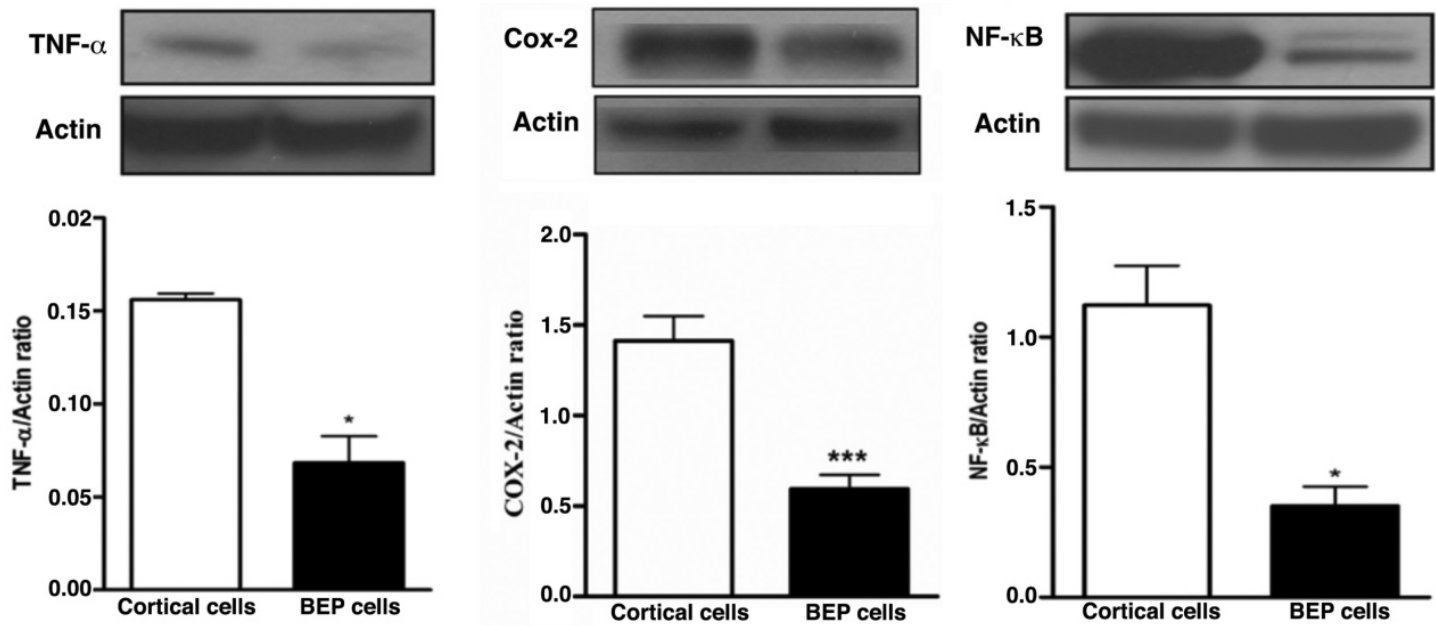

Figure 4. Effects of BEP neuron transplants in the hypothalamus on DMH-induced changes in inflammatory cytokines levels in the colon. (A-C) Western blot analysis of TNF- $\alpha(\mathbf{A}), \mathrm{COX}-2$ and $(\mathbf{B})$ and NF-KB $(\mathbf{C})$ of BEP and cortical neurons transplanted animals. Data are mean and SEM $(n=10)$. ** P<0.01 and $* * * \mathrm{P}<0.001$ between $\mathrm{BEP}$ cells and cortical cells transplanted groups.
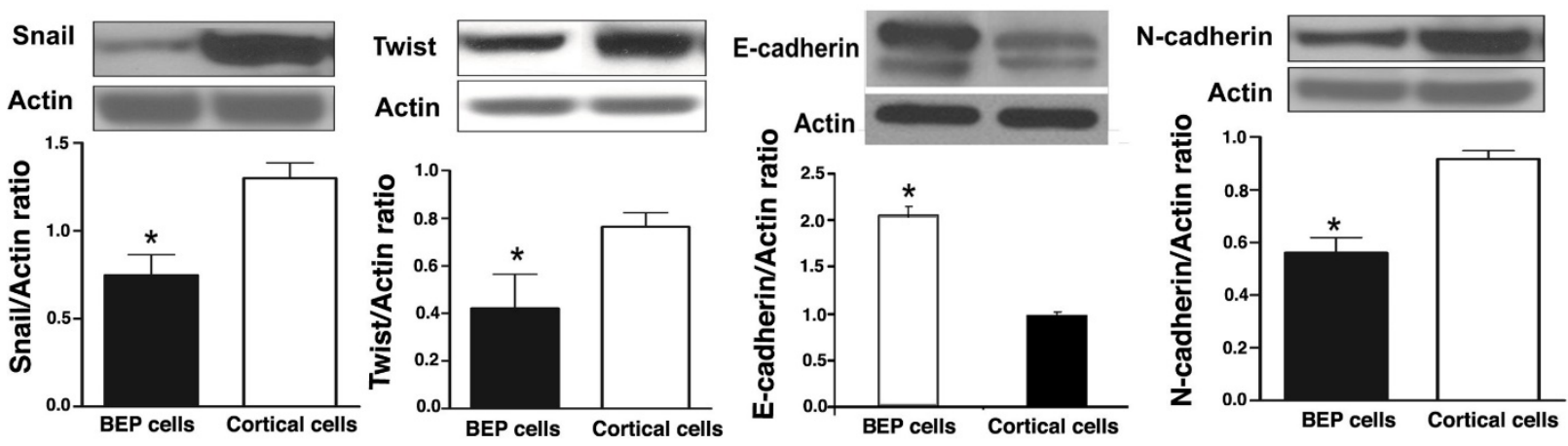

Figure 5. Effects of BEP neuron transplants in the hypothalamus on DMH-induced changes in EMT marker proteins levels in the colon. (A-D) The levels of Snail (A), Twist (B), E-cadherin (C) and N-cadherin (D) in colonic cancer tissues were detected by Western blotting. Data are mean and SEM ( $\mathrm{n}=10$ ). $* \mathrm{P}<0.05$ between BEP cells and cortical cells transplanted groups. 

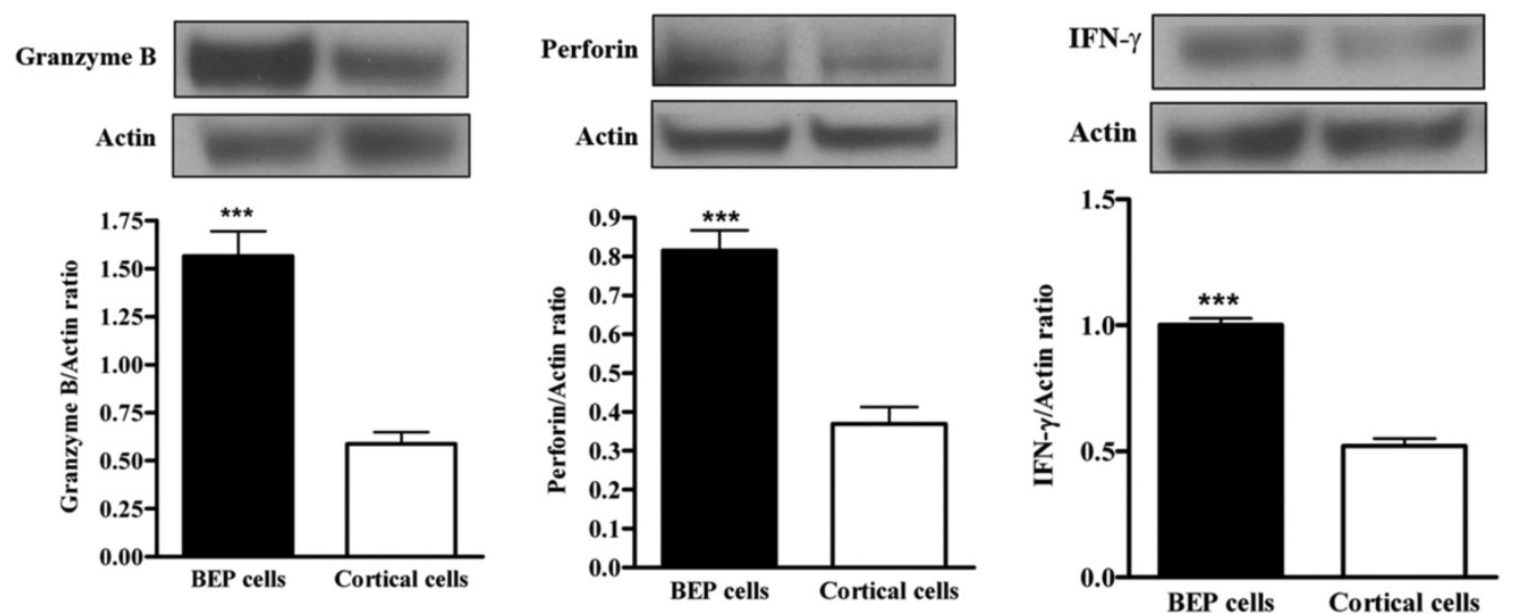

Figure 6. Effects of BEP neuron transplants in the hypothalamus on DMH-induced changes in NK cell cytolytic proteins levels in the colon. (A, C) The levels of granzyme (A), perforin (B) and IFN-Y (C) in spleen were detected by Western blotting. Data are mean and SEM $(n=10)$. ***P<0.001 between BEP cells and cortical cells transplanted groups.

Because beta-endorphin is naturally occurring within the human body, it is easier for individuals to engage in preventative behavior, by way of exercise or meditation, for example. Practicing maintaining an optimistic attitude can also be helpful. Stress prevention by way of beta-endorphin neuronal therapy may be useful as a tool for the management of cancer. Together, these findings suggest that BEP neurons in the hypothalamus have the ability to suppress colonic tumor formation possibly by influencing the ANS controls of innate immune functions in the liver.

We conclude that BEP neurons implantation might effectively block the inflammatory response and its further signaling. The transplants may also inhibit tumor growth and progression partly by repressing EMT. Thus, our observations provide new insights into the molecular mechanisms of inhibition of colorectal cancer by BEP neurons in the hypothalams.

\section{Acknowledgements}

This work is partly supported by a National Institute of Health grant R37AA08757, R21AA024330

\section{Competing Interests}

The authors have declared that no competing interest exists.

\section{References}

1. American Cancer Society. Cancer Facts \& Figures 2014. Atlanta: American Cancer Society; 2014

2. US Cancer Statistics Working Group. United States Cancer Statistics (USCS): 1999--2007 incidence and mortality data. Atlanta, GA: U.S. Department of Health and Human Services, CDC, National Cancer Institute; 2010. Available at http://www.cdc.gov/uscs. Accessed June 7, 2011.

3. Morin PJ, Sparks AB, Korinek V, Barker N, Clevers H, Vogelstein B, Kinzler $\mathrm{KW}$. Activation of beta-catenin-Tcf signaling in colon cancer by mutations in beta-catenin or APC. Science 1997; 275:1787-90.
4. Vasen HF. What is hereditary nonpolyposis colorectal cancer (HNPCC). Anticancer Res 1994; 14:1613-5.

5. Wynne O, Sarkar DK. Stress and neuroendocrine-immune interaction: a therapeutic role for $\beta$-endorphin. Kusnecov A and Anisman $\mathrm{H}$ (eds.) Handbook of Psychoneuroimmunology, Wiley Blackwell, Oxford, UK. 2013;::198-211.

6. Sarkar DK, Zhang C, Murugan S, Dokur M, Boyadjieva NI, Ortigüela M, Reuhl KR, Mojtehedzadeh S. Transplantation of $\beta$-endorphin neurons into the hypothalamus promotes immune function and restricts the growth and metastasis of mammary carcinoma. Cancer Res 2011 Oct 1;71(19):6282-91.

7. Zhang C, Murugan S, Boyadjieva N, Jabbar S, Shrivastava P, Sarkar DK. Beta-endorphin cell therapy for cancer prevention. Cancer Prev Res (Phila) 2015 Jan;8(1):56-67.

8. Murugan S, Boyadjieva N, Sarkar DK. Protective effects of hypothalamic beta-endorphin neurons against alcohol-induced liver injuries and liver cancers in rat animal models. Alcohol Clin Exp Res 2014 Dec;38(12):2988-97.

9. Sarkar DK, Boyadjieva NI, Chen CP, Ortigüela M, Reuhl K, Clement EM, Kuhn P, Marano J. Cyclic adenosine monophosphate differentiated beta-endorphin neurons promote immune function and prevent prostate cancer growth. Proc Natl Acad Sci USA 2008 Jul 1;105(26):9105-10.

10. Boyadjieva NI, Ortigüela M, Arjona A, Cheng X, Sarkar DK. Beta-endorphin neuronal cell transplant reduces corticotropin releasing hormone hyperresponse to lipopolysaccharide and eliminates natural killer cell functional deficiencies in fetal alcohol exposed rats. Alcohol Clin Exp Res 2009 May;33(5):931-7.

11. Sarkar DK, Murugan S, Zhang C, Boyadjieva N. Regulation of cancer progression by $\beta$-endorphin neuron. Cancer Res 2012 Feb 15;72(4):836-40.

12. Sarkar DK, Zhang C. Beta-endorphin neuron regulates stress response and innate immunity to prevent breast cancer growth and progression. Vitam Horm 2013; 93:263-76.

13. Maskens AP. Histogenesis and growth pattern of 1,2-dimethylhydrazine induced rat colon adenocarcinoma. Cancer Res 1976; 36:1585-92.

14. Fiala ES. Investigations into the metabolism and mode of action of the colon carcinogens 1,2-dimethylhydrazine and azoxymethane. Cancer 1977; 40:2436-45.

15. Reddy BS, Narisawa T, Maronpot R, Weisburger JH, Wynder EL. Animal models for the study of dietary factors and cancer of the large bowel. Cancer Res 1975; 35:3421-6.

16. Bird RP. Role of aberrant crypt foci in understanding the pathogenesis of colon cancer. Cancer Lett 1995; 93:55-71.

17. Shamsuddin AKM, Trump BF. Colon epithelium I. Light microscopic, histochemical and ultrastructural features of normal colon epithelium of male Fischer 344 rats. J Nat Cancer Inst 1981; 66:37588.

18. Cheng L, Lai MD. Aberrant crypt foci as microscopic precursors of colorectal cancer. World J Gastroenterol 2003; 9:2642-9.

19. Sugimura T. Multistep carcinogenesis: a 1992 perspective. Science 1992; 258:603-7.

20. Takahashi H, Hosono K, Endo H, Nakajima A. Colon epithelial proliferation and carcinogenesis in diet-induced obesity. J Gastroenterol Hepatol 2013 Dec;28 Suppl 4:41-7.

21. Hao XP, Pretlow TG, Rao JS, Pretlow TP. Beta-catenin expression is altered in human colonic aberrant crypt foci. Cancer Res 2001; 61:8085-8.

22. Janakiram NB, Rao CV. The role of inflammation in colon cancer. Adv Exp Med Biol 2014; 816:25-52.

23. Rakoff-Nahoum S. Why Cancer and Inflammation? Yale J Biol Med 2006; 79:123-130. 
24. Francuz T, Czajka-Francuz P, Cison-Jurek S, Wojnar J. [The role of inflammation in colon cancer pathogenesis]. Postepy Hig Med Dosw (Online). 2016 Apr 27; 70:360-6.

25. Pesic M, Greten FR. Inflammation and cancer: tissue regeneration gone awry. Curr Opin Cell Biol. 2016 Dec; 43:55-61.

26. Kalluri R, Weinberg RA. The basics of epithelial-mesenchymal transition. J Clin Invest 2009; 119:1420-1428.

27. Cano A, Perez-Moreno MA, Rodrigo I, Annamaria L, Blanco MJ, et al. The transcription factor Snail controls epithelial-mesenchymal transitions by repressing E-cadherin expression. Nature Cell Biol 2000; 2:76-83.

28. Zhu QC, Gao RY, Wu W, Qin HL. Epithelial-mesenchymal transition and its role in the pathogenesis of colorectal cancer. Asian Pac J Cancer Prev 2013;14(5):2689-98.

29. Berraondo P, Minute L, Ajona D, Corrales L, Melero I, Pio R. Innate immune mediators in cancer: between defense and resistance. Immunol Rev 2016 Nov;274(1):290-306.

30. Guillerey C, Huntington ND, Smyth MJ. Targeting natural killer cells in cancer immunotherapy. Nat Immunol 2016 Aug 19;17(9):1025-36.

31. Vesely MD, Kershaw MH, Schreiber RD, Smyth MJ. Natural Innate and Adaptive Immunity to Cancer. Annu Rev Immunol 2011; 29:235-271.

32. Liu Y, Zeng G. Cancer and Innate Immune System Interactions: Translational Potentials for Cancer Immunotherapy. J Immunother 2012; 35:299-308.

33. Segerstrom SC, Miller GE. Psychological Stress and the Human Immune System: A Meta-Analytic Study of 30 Years of Inquiry. Psychol Bull 2004; 130:601-630.

34. Thaker PH, Sood AK. The Neuroendocrine Impact of Chronic Stress on Cancer. Semin Cancer Biol 2008; 18:164-170.

35. Mariani F, Sena P, Roncucci L. Inflammatory pathways in the early steps of colorectal cancer development. World J Gastroenterol 2014 Aug $7 ; 20(29): 9716-31$ 